Supporting Information

\title{
Zeta-Potential Dependent Self-Assembly For Very Large Area Nanosphere Lithography
}

\author{
Gabriel Cossio ${ }^{1}$, Edward T. Yu ${ }^{1}$
}

${ }^{1}$ Department of Electrical and Computer Engineering, The University of Texas at Austin,

\author{
Austin, Texas, 78758, United States
}


Supplementary Table S1: Zeta potential of $200 \mathrm{~nm}$ polystyrene NS solutions after $\mathrm{NaCl}$ addition which appear in figure $3 \mathrm{c}$ of the main text.

\begin{tabular}{|l|c|c|c|c|}
\hline $\mathrm{NaCl}(\mathrm{M})$ & 0 & $1 \mathrm{E}-3$ & $1 \mathrm{E}-1$ & $5 \mathrm{E}-1$ \\
\hline $\begin{array}{l}\text { Zeta } \\
\begin{array}{l}\text { Potential } \\
\text { Magnitude } \\
(\mathrm{mV})\end{array}\end{array}$ & 71.5 & 52.7 & 40.9 & 25.6 \\
\hline
\end{tabular}


Supplementary Figure 1 (S1): (Top) Image of four successive $200 \mathrm{~nm}$ polystyrene monolayer growths and depositions on 5"x 5" flexible PET substrates. Monolayers appear white on the PET film. Shading that appears in the sheets are due to variations in the background that are being reflected. (Bottom) For clarity a close-up image is shown of a 5" 5 " PET sheet coated in a $200 \mathrm{~nm}$ polystyrene monolayer. As can be seen monolayer growth is highly uniform. A large defect appears in the top left corner of the sheet due to transferring of the monolayer to the PET sheet which can result in 

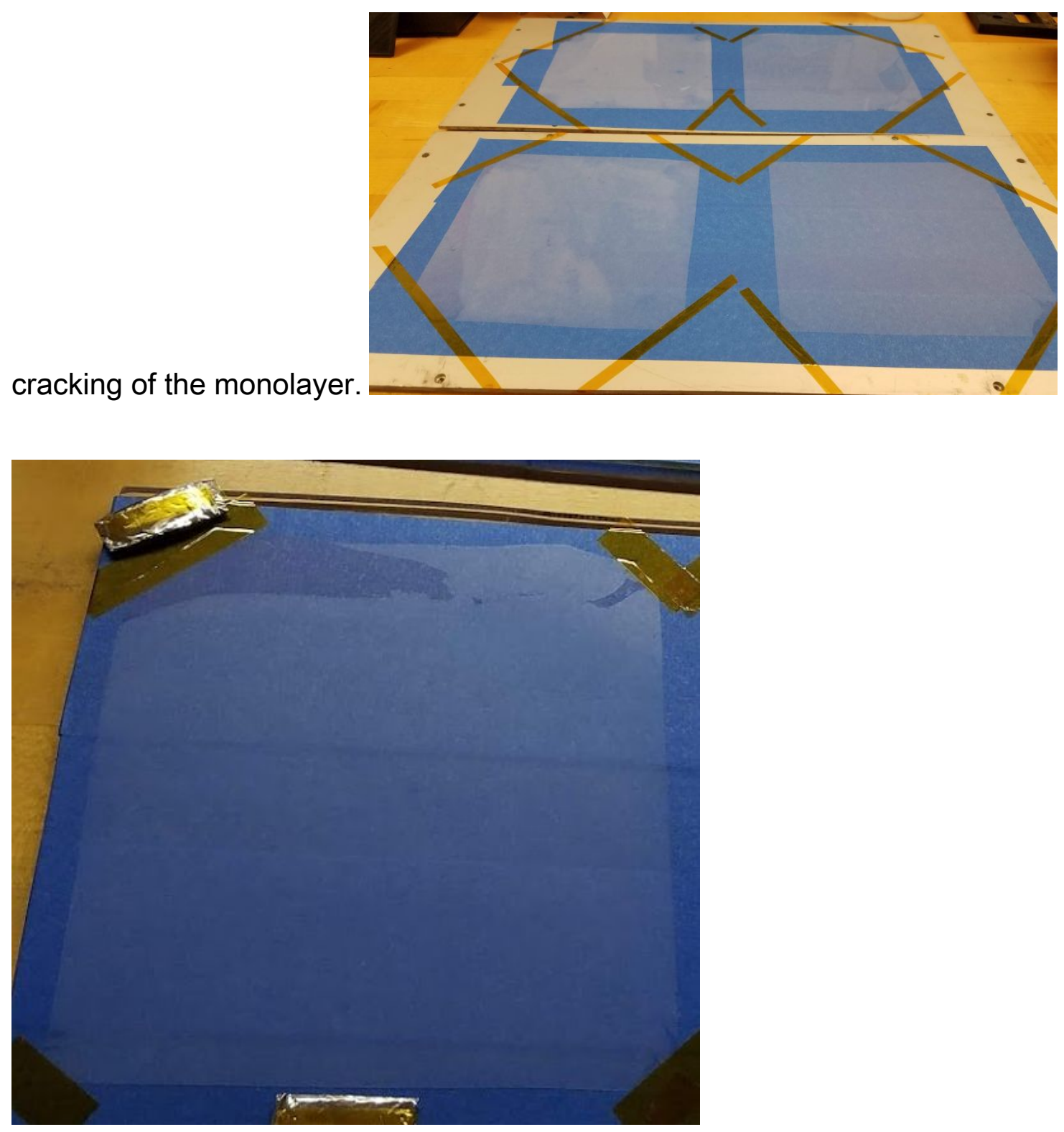

Supplementary Figure 2 (S2): Images of $2 \mu \mathrm{m}$ polystyrene NS self-assembled monolayer growth with and without $1 x$ solvent washing procedure in $100 \%$ ethanol. NS solutions were injected for 20 minutes for both trials. Injection parameters and solvent washing procedures can be found in the Experimental Methods section of the 
Supporting Information.

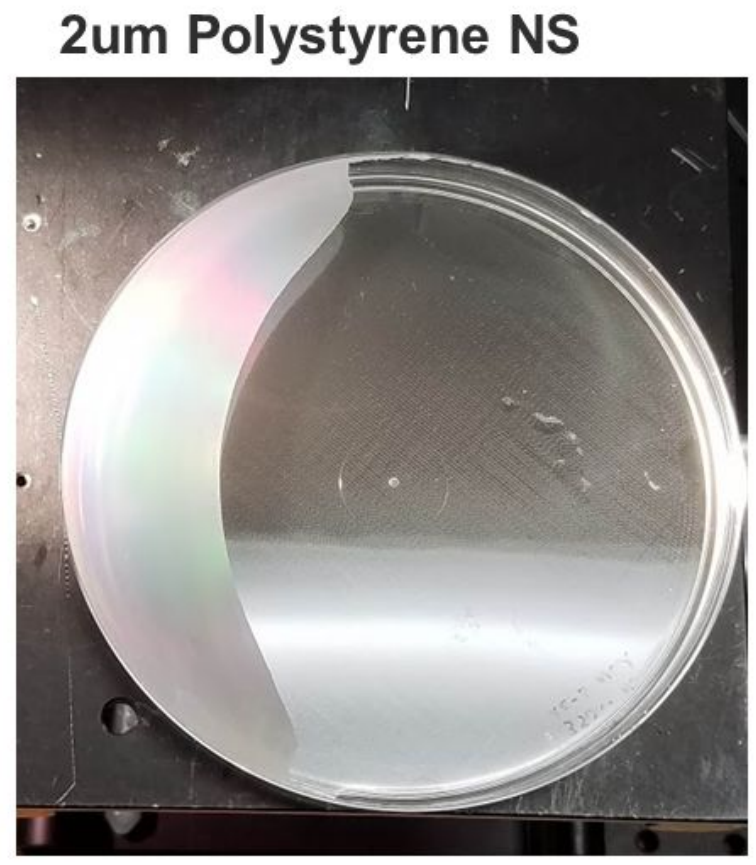

No zeta potential modulation 2um Polystyrene NS

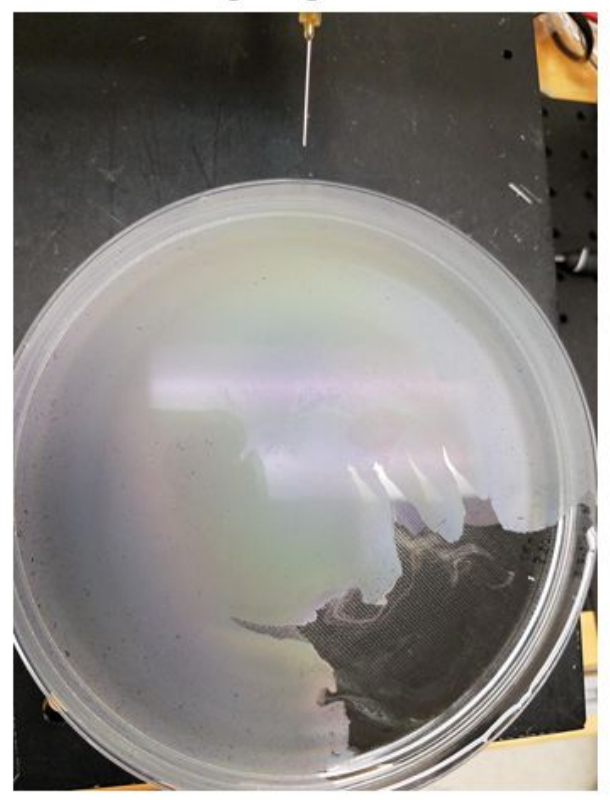

1x EtOH solvent washing

Supplementary Note 1: Addition of Cationic Surfactant to Silica NS for Self-Assembly 
Silica NSs, like SDS terminated polystyrene NSs, have a large negative zeta potential and are very stable in aqueous solutions. However, the stability of colloidal silica NS's arises from the negatively charged surface of the silica particles that increases hydrophilicity and prevents flocculation. As described in the main text, the strong hydrophilicity of the particles would not be conducive to efficient monolayer selfassembly. Despite this, by adding a cationic surfactant (CTAB) to the silica NS's we can lower the zeta potential magnitude of the solution from $61 \mathrm{mV}$ to $41 \mathrm{mV}$ and enable large monolayer growth. No discernable $\mathrm{SiO}_{2} \mathrm{NS}$ monolayer self-assembly was observed without the addition of CTAB. The cationic head adsorbs to the negative silica surface which leaves the hydrophobic tail protruding from the nanoparticle surface. By increasing the concentration of cationic surfactant adsorbed onto the silica surface, the stability of the silica NS in an aqueous solution can be reduced. The reduced stability of the silica NS's increases the proximity between the silica NS's and facilitates selfassembly. This is particularly interesting as no silica NS monolayers were seen to be formed on the air water interface via the injection method without the addition of CTAB. Also, the density of silica NS's are $\sim 2 x$ that of polystyrene NS's and by varying only the 
zeta potential magnitude we can form NS's monolayers irrespective of density, material, and size. These results further support the conclusion that the zeta-potential of the injecting solution is the key variable in ensuring successful self-assembly with this method.

Supplementary Figure 3 (S3): (a) SEM image of self-assembled monolayer of $2 \mu \mathrm{m}$ polystyrene NSs. Scale bar is $4 \mu \mathrm{m}$. (b) SEM image of self-assembled monolayer of 200 $\mathrm{nm}$ silica NSs. Scale bare is $2 \mu \mathrm{m}$. Self-assembled monolayers of $200 \mathrm{~nm}$ polystyrene NSs are shown in figure $5 f$ of the main text. 

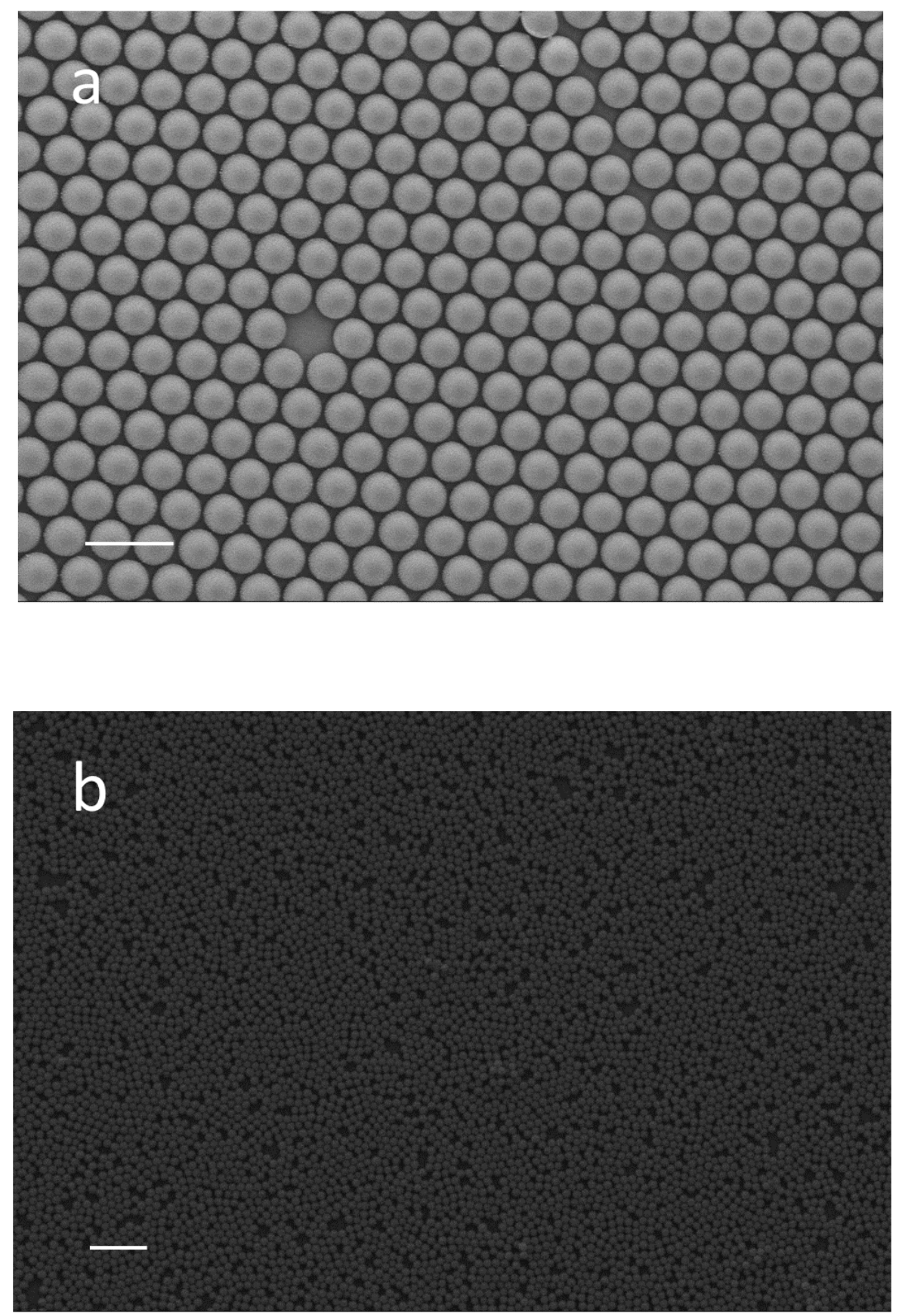

Supplementary Figure 4 (S4): Photograph of three PET sheets. (a) PET sheet with moth-eye antireflection nanopatterning as described in figure 5 of the main text. The antireflection nanopatterning was successfully patterned inside the area enclosed by the 
dashed red line. The nanopatterned area appears closer in color to the background which occurs due to the increased transmission and decreased reflection from the moth-eye nanopatterned surface. The non-nanopatterned region, on the right edge of the samples, appears brighter due to more prominent glare from specular and diffuse reflections. These defects occur when the monolayer does not adhere to the substrate during the draining and deposition steps of the transfer (figure 5a,b) (b) PET sheet with no nanotexturing. Strong reflections are prominent across the entire sample surface, similar to the nonpatterned regions of $(a, c)$. (c) PET sheet with moth-eye nanopatterning. The moth-eye patterned are was not successfully formed on the left and top edges of the sample. An outline is left out so that entire sample can be seen. Similar to (a), the background image in the moth-eye textured regions appears clearer due to a decrease in reflections. Similar to (b), the defected area on the left and top edge have a more prominent glare due to enhanced reflection. 


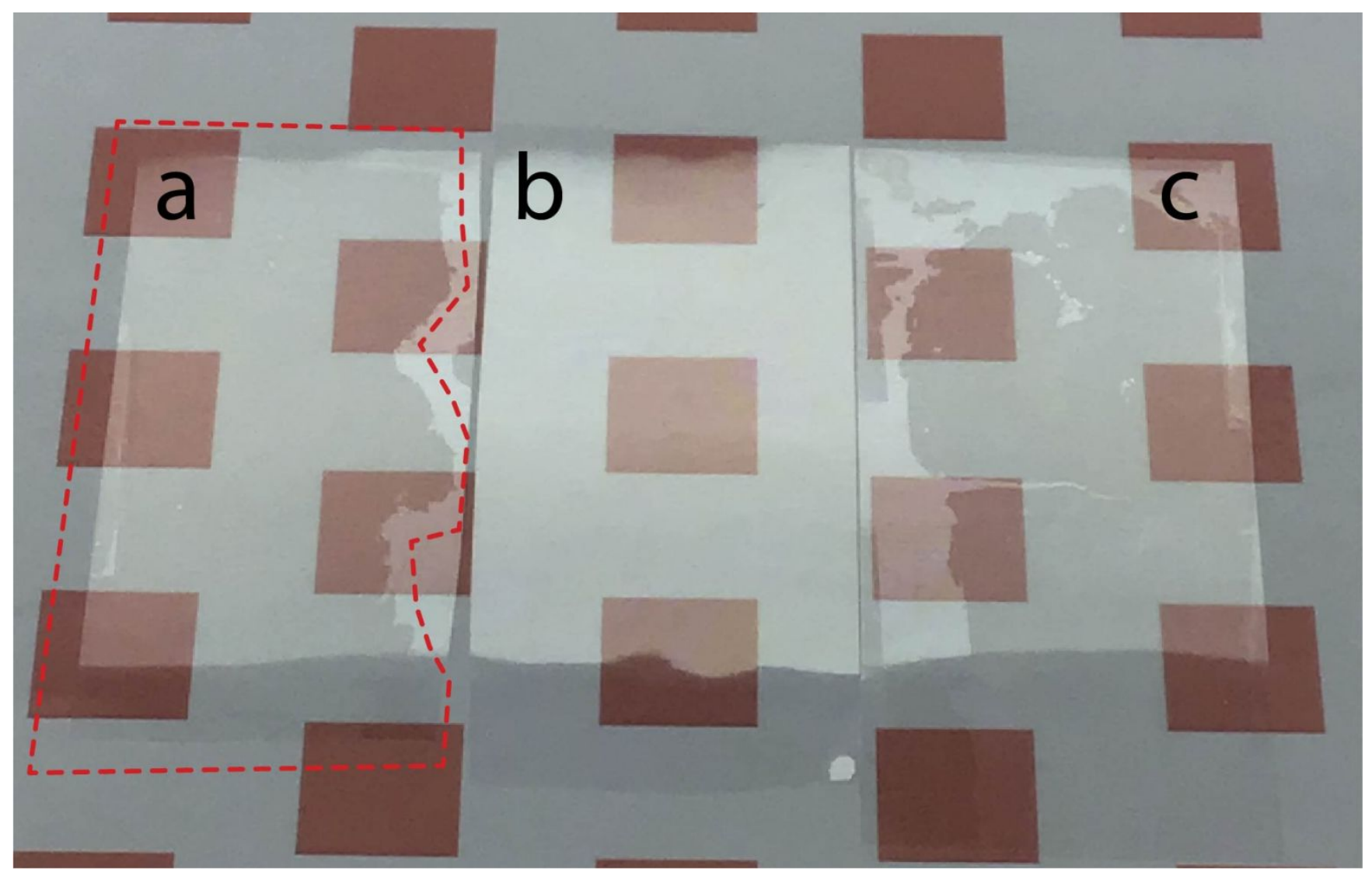




\section{Experimental Methods}

\section{Preparation of Nanosphere Solution Via Solvent Washing}

$200 \mathrm{~nm}$ and $2 \mu \mathrm{m}$ Polystyrene NS solutions (2.5\% weight/volume) were purchased from

Polysciences Inc. These spheres are terminated with anionic sodium dodecyl sulfate

(SDS) surfactant molecules. A reference solution was made by first centrifuging the

commercial solution at 14,000 RPM for 22 minutes until pellets were formed. The

supernatant was then removed and an equal volume of milliQ DI water was used to

replace the original solvent. Sonication and a vortex mixer were then used to resuspend

the NS. This was done in order to remove any unspecified buffers, surfactants, or other

contaminants remaining in the original solution from the commercial NS fabrication

process. Test solutions were made from the resulting "cleaned" solution. First, our

"cleaned" solution was centrifuged, pelleted, and the supernatant volume was decanted. 
An equal volume of either ethanol, isopropanol, or acetone was then added, and the pellet was resuspended via vortexing and sonication. Concentrations of ethanol, isopropanol, or acetone ranging from $0-100 \%$ were used. After resuspending in solvent, the solution was again pelleted via centrifugation and the supernatant was removed and replaced with an equal volume of milliQ DI water. The pelleting of the solvent washed NSs required careful optimization that yielded the centrifugation parameters given above. If centrifugal forces are too high or centrifugation is done for too long irreversible aggregation of the pellet may occur due to the increased van der Waal forces between NSs. One final cycle of pelleting and resuspension in milliQ DI water was completed in order to ensure that milliQ DI water was the only solvent present in the final solution.

\section{Preparation of Nanosphere Solution Via Addition of $\mathrm{NaCl}$}

A second set of test solutions was made by dissolving different quantities of $\mathrm{NaCl}$ in the "cleaned" NS solution. Unlike the solvent washing method, which alters the polarity of the solution and therefore changes the density of SDS molecules on the NS surface, 
this method affects the zeta potential by adding ionic charges to the solution which act to change the solution's ionic strength of the solution. With increasing ionic strength, the repulsive SDS shell around the NS's can be screened and the zeta potential can be lowered.

\section{Preparation of Injected Nanosphere Solution}

Both polystyrene NS solutions prepared by $\mathrm{NaCl}$ addition or by solvent washing were mixed in a 1:2 ratio with 200 proof ethanol. These solutions are used in testing the nanosphere self-assembly with ethanol as the convective spreading agent.

\section{Preparation of Silica NS Solution}

$200 \mathrm{~nm}$ silica NS solutions (10\% weight/volume) were purchased from Polysciences

Inc. The commercial solution was centrifuged at 4,000 RPM for 10 minutes until pellets were formed. The supernatant was then removed and an equal volume of milliQ DI 
water was used to replace the original solvent. Sonication and a vortex mixer were then used to resuspend the NS. This was done in order to remove any unspecified buffers, surfactants, or other contaminants remaining in the original solution from the commercial NS fabrication process. $10 \mu \mathrm{L}$ of hexadecyltrimethylammonium bromide $(0.0275 \mathrm{M})$ was added to $0.5 \mathrm{~mL}$ of silica NS solution and vortexed for 2 minutes. An equal volume of ethanol was added to make a 1:1 solution of ethanol to silica NS solution.

\section{Injection Apparatus.}

A commercial syringe pump was used to drive the colloidal solution through the injection system. A hypodermic needle (25 gauge) was attached to a servo motor to accurately determine the injection angle (contact angle between needle and water). This needle is connected to a servo motor and micrometer translation stage to enable accurate control and measurement of both the injection angle $(\theta)$ and the position of the needle above the air-water interface $(\mathrm{H})$. The angular position and meniscus heights are resolved to 
within $1^{\circ}$ and $25 \mu \mathrm{m}$, respectively. Injection widths $(\mathrm{w})$ are controlled by the choice of needle gauge. The NS solution exits the needle tip and enters a meniscus formed between the needle and the water surface, as shown in Figure 2c-2e. All injections are performed with a $15^{\circ}$ contact angle $(\theta), 22.88 \mathrm{~mL} / \mathrm{hr}$ flow rate, $0.635 \mathrm{~mm}$ meniscus height $(\mathrm{H})$, and $0.250 \mathrm{~mm}$ injection width $(\mathrm{w})$ as defined in figure $2 \mathrm{~b}$ of the main text. The monolayer growth rate increases with flow rate, and the overall injection parameters were chosen such that the monolayer growth rate was maximized for injections with the highest magnitude zeta potential.

\section{Zeta potential measurements}

The zeta potential of polystyrene and silica spheres was measured using a Malvern

Zetasizer Nano ZS. All reported zeta potential measurements were done with a $0.037 \%$ $(w / v)$ concentration of NSs. 
Reflectance was measured using a single grating monochromator Optronics

Laboratories OL 750 platform. Specular reflectance measurements were completed with a gonioreflectance attachment (OL 750-75MA) and diffuse measurements with a integrating sphere diffuse reflectance attachment (OL-740-70). 